\title{
GI-MS48-P07 | BENEFITS OF TRANSMISSION MODE IN XRD
}

Berthier, Eric (Thermo Fisher Scientific, Artenay, FRA); Pillière, Henry (Thermo Fisher Scientific, Artenay, FRA)

Powder X-ray diffraction (XRD) is an analytical technique which offer a wide range of instrumental configuration, in order to respond to specific structural characterization. The most frequent setup is based on the irradiation of the sample surface by an incident beam, to allow sample the surface emission of the diffracted signal (Bragg-Brentano or asymmetric geometry). However, diffraction signal can also be obtained by transmission, as described by the Debye-Scherrer geometry. Because of its original instrumental configuration, ARL EQUINOX X-Ray diffractometers allow to perform both setups, and allow to get complementary information on the sample itself. This is the case for bi-dimensional structures like clays, graphite family, etc. For any crystallized material, transmission mode always offers a higher resolution signal, because there is a low impact of the incident angle. Preferred orientation is also minimized, and quantitative analysis is often better accuracy. From a reactional point of view, transmission allow to record sample under a certain conditioning (atmosphere, in a liquid, to avoid reactivity), at room conditions or by applying temperature or pressure. We proposed to show some examples explaining the benefit of transmission mode in XRD. 